\title{
X-ray emission near the substellar limit: The $\sigma$ Orionis and Taurus star forming regions
}

\author{
F. Mokler and B. Stelzer
}

\begin{abstract}
Max-Planck-Institut für extraterrestrische Physik, Postfach 1312, 85741 Garching, Germany
Received 19 April 2002 / Accepted 11 June 2002

Abstract. We have carried out an extensive search for X-ray emission from young, very low-mass objects near and beyond the substellar limit, making use of archived ROSAT PSPC and HRI observations pointed at Brown Dwarfs and Brown Dwarf candidates in the young $\sigma$ Orionis and Taurus-Auriga associations. In $\sigma$ Ori we identify three Brown Dwarf candidates with X-ray sources; in Taurus-Auriga we add one further X-ray detection of a Brown Dwarf to the list published earlier. We combine this data with all previously X-ray detected Brown Dwarfs and Brown Dwarf candidates in young stellar associations and star forming regions to perform a study of stellar activity parameters on the as yet largest sample of young, very low mass objects. A similar relation between X-ray and bolometric luminosity, and $\mathrm{H} \alpha$ emission, respectively, as is known for $\mathrm{T}$ Tauri stars seems to hold for young objects down to the substellar limit, too. No signs for a change in X-ray activity are found on the transition to substellar masses.
\end{abstract}

Key words. X-rays: stars - stars: formation, low-mass, Brown Dwarfs, coronae, activity

\section{Introduction}

Late-type stars exhibit strong signs of magnetic activity such as $\mathrm{H} \alpha$, Ca II or X-ray emission from hot thermal plasma confined in magnetic fields on the star. For fully convective stars (with spectral type $\sim \mathrm{M} 3$ and later) the change in interior structure is expected to result in a change of the field sustaining dynamo and, therefore, of the emission properties. However, no clear change in common activity diagnostics is found at these spectral types. Gizis et al. (2000), Basri (2001) and Mohanty \& Basri (2002) have observed a decline in $\mathrm{H} \alpha$ emission for old (>1 Gyr) L-type objects in the field, suggesting that a decline in dynamo activity sets in beyond the boundary where the objects become fully convective and close to the borderline to substellar masses. An X-ray study of K- and early M-stars within the solar neighborhood by Fleming et al. (1995) did not unveil any change in X-ray activity for objects on the transition towards fully convective energy transport. It is unclear to date how activity in fully convective and substellar objects depends on parameters such as rotation or age. Studying these relations is essential to an understanding of the underlying dynamo mechanism.

An important measure for magnetic activity on late-type stars of all ages is X-ray emission which is usually explained by emission from a hot plasma. Within the last few years, $\mathrm{X}$-ray emission has also been detected from Brown Dwarfs (BD) and BD Candidates (see e.g. Neuhäuser \& Comerón 1998; Neuhäuser et al 1999). In these early studies with ROSAT only BDs in the Chamaeleon, Taurus and $\rho$ Ophiuchus star

Send offprint requests to: F. Mokler,

e-mail: fmokler@mpe.mpg.de forming regions were detected, while all older substellar objects in stellar associations such as the Pleiades and in the field are X-ray quiet down to the ROSAT detection limit. More recent observations with Chandra revealed X-ray emission from further young BDs in Orion (Garmire et al. 2000; Feigelson et al. 2002), $\rho$ Oph (Imanishi et al. 2001), and IC 348 (Preibisch \& Zinnecker 2001; Preibisch \& Zinnecker 2002). To date only one old field BD is known to emit X-rays, namely LP 944-20 which was detected only during a flare (Rutledge et al. 2000). Here, we extend the investigations with a study of X-ray emission from the very low-mass (VLM) members in the $\sigma$ Orionis association, and an update of the $\mathrm{X}$-ray emitting BD population in Taurus.

The $\sigma$ Ori star forming region was discovered by Walter (1997), and was shown to be rich in X-ray sources. Photometric and spectroscopic observations by Béjar et al. (1999), Zapatero et al. (2000), and Béjar et al. (2001) have revealed $\sim 80$ objects in the low mass regime close to and below the hydrogen burning mass limit (HBML) in the OB1b association near the multiple star $\sigma$ Orionis at a mean distance modulus of $D M=7.73$ measured by HIPPARCOS, corresponding to $352 \mathrm{pc}$ (Béjar et al. 1999). The age of this cluster is estimated to be $1-7 \mathrm{Myrs}$ where the upper age limit is given by constraints of the central star $\sigma$ Orionis: being of spectral type 09.5 and still in the hydrogen burning phase, it cannot be older than 5-7 Myrs (see Béjar et al. 2001; Barrado y Navascues et al. 2001, and references therein). According to Béjar et al. (2001), the position of all members in the H-R diagram is best compatible with an isochrone for 5 Myrs. Based on measurements of lithium abundances in $\sigma$ Ori members Zapatero et al. (2002) give as most likely age 2-4 Myrs with an upper limit of 8 Myrs. 
At this young age, even VLM objects are still very luminous. In addition, the $\sigma$ Ori association is hosted in a region of very low extinction $\left(E_{B-V}=0.05\right.$; Lee 1968). This provides excellent conditions to study activity in faint VLM objects despite the considerable distance to the cluster.

Taurus-Auriga is one of the nearest ( $d=140 \mathrm{pc}$; Elias 1978) and most-studied regions of star formation. The X-ray emission from late-type pre-main sequence stars in Taurus-Auriga was recently discussed by Stelzer \& Neuhäuser (2001) (hereafter SN01). SN01, whilst concentrating on the G- to early M-type $\mathrm{T}$ Tauri Stars, put forth the ROSAT PSPC detection of several objects with spectral types beyond M5, i.e. near the substellar limit. Four additional BDs have been discovered in that region (Martín et al. 2001b) since. In this paper we present a detailed analysis of the X-ray activity of all VLM objects in Taurus-Auriga including the new BDs. In the following we used the term "very-low mass" for objects with spectral type later than M4.

In Sect. 2, we outline the criteria we used when selecting our data samples. In Sect. 3, we describe the ROSAT data analysis. We present the results for both $\sigma$ Orionis and Taurus in Sect. 4. In Sect. 5, we perform a comparative study of the X-ray properties of all young BDs and BD candidates detected so far, including tests for variability and an investigation of correlations with other activity parameters. A summary of our results is given in Sect. 6 .

\section{Sample selection}

As we searched for X-ray emission from VLM objects in the $\sigma$ Orionis and Taurus star forming regions our sample consists of all objects with spectral type $\sim \mathrm{M} 5$ and later. At an age of 5 Myrs all objects with spectral type M7 and later can be considered to be substellar (Basri 2000 and references therein). Therefore, our sample includes the transition from VLM stars to BDs.

For about one third of the VLM $\sigma$ Ori members the spectral types are known, and cover the range from M4.5 to L6 (Béjar et al. 1999; Béjar et al. 2001; Martín et al. 2001a and Barrado y Navascues et al. 2001). A large fraction of the cluster members, however, has been observed only photometrically, so far. For the X-ray detected objects we estimated the spectral types by comparing their $I-J, I-K$ and $R-I$ colors with those of the objects in $\sigma$ Ori with known spectral type, assuming negligible extinction.

The Taurus sample consists of all objects beyond spectral type M4 included in SN01, and the newly identified BDs from Martín et al. (2001b).

\section{Observations and data analysis}

We systematically searched the ROSAT archive for deep observations including VLM objects in Tau and $\sigma$ Ori. The majority of ROSAT PSPC observations with BDs or BD candidates in Taurus can be found in SN01. We added to this list all PSPC pointings including any of the objects from Martín et al. (2001b), i.e. ROSAT Observation IDs 201016p and 201017p. Besides we examined all HRI pointings with
Taurus objects from our sample (202156h, 202031h, 201090h, 201046h, 201623h-1/-2, 201089h, 201617h/-1).

Eight PSPC pointings are available for the $\sigma$ Orionis region: 180023p-0/-1, 200198p, 200932p, 201151p-0/-1, 900198p, and 900386p. Careful inspection showed that only pointings $201151 \mathrm{p}-0$ and $201151 \mathrm{p}-1$ are useful for aiming at faint detections. In the remaining cases, the objects are either located at a large off-axis angle $\left(\Delta \geq 39^{\prime}\right)$ within the field of view (FOV), or the net exposure time at the position of the objects is too low as it was covered by the ribs of the telescope over a substantial fraction of the exposure time. Hence, we exclusively used pointings 201151 p- 0 and 201151p-1 from this list. In order to improve the sensitivity both pointings were merged to add up to one pointing with total exposure time of $\sim 33 \mathrm{ksec}$. In the following we refer to this combined observation as 201151p. All available 35 HRI pointings in $\sigma$ Ori are centered at the same sky position as 201151p. They were all added up to yield a pointing with total exposure time of $\sim 81 \mathrm{ksec}$. This pointing will be named after the last one of the whole sequence, $201915 \mathrm{~h}$.

The data were analysed in the MIDAS/EXSAS environment. We used a combination of the map, local and maximum likelihood detection algorithm described by Cruddace et al. (1987). The source detection threshold was set to $M L \geq 7.4$ (PSPC) and $M L \geq 5.0$ (HRI), respectively (corresponding to a reliability $>99 \%$; see Neuhäuser et al. 1995). Source detection was performed in the broad $(0.1$ to $2.0 \mathrm{keV})$, soft $(0.1$ to $0.4 \mathrm{keV})$, hard $1(0.5$ to $0.9 \mathrm{keV})$ and hard $2(0.9$ to $2.0 \mathrm{keV})$ energy bands of the PSPC, and in the broad band ( 0.1 to $2.0 \mathrm{keV})$ of the HRI. In order to get qualitative information on the X-ray spectrum, we calculated the PSPC hardness ratios, $H R 1$ and $H R 2$, from the X-ray count rates in the soft, hard 1 and hard 2 band:

$H R 1=\frac{Z_{\mathrm{h} 1}+Z_{\mathrm{h} 2}-Z_{\mathrm{s}}}{Z_{\mathrm{h} 1}+Z_{\mathrm{h} 2}+Z_{\mathrm{s}}}$

and

$H R 2=\frac{Z_{\mathrm{h} 2}-Z_{\mathrm{h} 1}}{Z_{\mathrm{h} 2}+Z_{\mathrm{h} 1}}$

where $Z_{\mathrm{x}}$ denotes the count rate in the corresponding energy band.

For the identification of a $\mathrm{BD}$ or $\mathrm{BD}$ candidate with an $X$-ray source we allowed for a maximum offset of $30^{\prime \prime}$ in the PSPC pointing and $10^{\prime \prime}$ in the HRI pointing, for sources in the inner area of the FOV, i.e. at off-axis angles smaller than $30^{\prime}$ (PSPC) and 6' (HRI), corresponding to the spatial resolution of the respective detectors. For X-ray sources at larger offaxis angles we gradually increased the identification radius as described by SN01 to take account of the degradation of the PSPC point spread function (PSF). The HRI data were treated in an analogous way. Visual inspection of the X-ray images showed that the reliability of automatic source detection is limited for faint X-ray sources in the immediate neighborhood of a bright one. Therefore, we performed an additional check of the ROSAT images by eye. In the case of S Ori 03, a faint X-ray source can be clearly discerned in the PSPC pointing. However, it was not found by the detection procedure. For this source we extracted the photons from a circular region centered on the optical position of S Ori 03 (see Table 2). 
For the photon extraction radius, we used the $99 \%$ quantile radius of the point spread function at $1 \mathrm{keV}$.

When converting the X-ray count rates into fluxes, we assumed a Raymond-Smith spectrum representing a thermal plasma at $1 \mathrm{keV}$. In contrast to the analysis of SN01 we did not attempt to split the $\mathrm{X}$-ray counts among the components in binary systems as the multiplicity of most objects in our sample is unknown.

\section{X-ray identifications in $\sigma$ Orionis and Taurus}

Following the procedure described in the previous section we detected X-ray sources near seven VLM $\sigma$ Ori members and 13 BD candidates and BDs in Taurus. We double-checked with the SIMBAD and GSC catalogues for other possible counterparts to these X-ray sources within a radius of $30^{\prime \prime}$. In ambiguous cases, we also consulted the Digitized Sky Survey (DSS). Below we describe the results for the individual objects.

\section{1. $\sigma$ Orionis}

With Table 1 we provide a list of all VLM $\sigma$ Ori members inside the error box of a ROSAT detection. In the cases of S Ori 07 and S Ori 43 , we found catalogued objects whose optical positions are closer to the X-ray position than that of the $\sigma$ Ori members. The GSC and SIMBAD object closest to S Ori 07 is Haro 5-19, an emission line star, with an offset of 2.3" from the X-ray position, whereas the offset of S Ori 07 is $9.9^{\prime \prime}$. Hence, the X-ray emission probably originates from the Haro star. The closest counterpart to the X-ray source near S Ori 43 is the GSC object 12906026 with an offset of $2.2^{\prime \prime}$, whereas the offset between S Ori 43 and the X-ray source is 4.1" (PSPC) and 3.9" (HRI), respectively. Checking the DSS image we conclude from the relative position of the X-ray source, the GSC object and S Ori 43 that the X-ray source is more likely to be assigned to the GSC source than to S Ori 43. For the remaining five X-ray identified $\sigma$ Ori members, no other counterparts neither in SIMBAD nor in the GSC were found. However, the offsets of S Ori 08 and S Ori 51 with respect to the X-ray position are near the limit of the ROSAT error box. At the position of the X-ray source near S Ori 51 an unknown optical object can clearly be distinguished on the DSS image. For S Ori 08, there is an optical source discernable both at its optical position and at the position of the nearby X-ray source. Therefore, we assign the X-ray emission to the DSS object at the same position in both cases. The identification of S Ori 03 with the X-ray source is unequivocal from the inspection of the DSS images. In the cases of S Ori J053926.8 and S Ori J053948.1 no other clear counterpart was found in the DSS either. In the following we discuss only these three unambiguous detections. For the other cases, where the nature of the additional optical counterparts is not known, we plan follow-up spectroscopy.

In Table 2 we list the X-ray properties of the three ROSAT detections which were clearly identified with $\sigma$ Ori members. All of them were detected in the PSPC pointing, and one object, S Ori 03, was also detected in the HRI pointing. In Fig. 1 we display the portion of the PSPC and HRI images that include these $\sigma$ Ori detections.

\subsection{Taurus}

The study by SN01 showed that about half of the BD candidates in the Taurus region known at that time were detected with the PSPC in pointed observations. Their X-ray luminosities are in agreement with the earlier study by Neuhäuser et al. (1999). We adopted the values listed in SN01, and added an analysis of HRI observations of the Taurus sample as described in Sect. 2. This analysis resulted in one additional HRIdetection of the M5-type object, V410x-ray5a, which had already been detected during a PSPC observation.

Furthermore, we searched all PSPC and HRI observations for X-ray emission from the position of the BDs newly discovered by Martín et al. (2001b), and found that one of them, CFHT-BD-Tau 4 , is detected during a $\sim 10 \mathrm{ksec}$ PSPC pointing. Cross-correlation with the GSC and SIMBAD as well as visual inspection of the corresponding DSS images showed that in all cases the X-ray emitter can clearly be identified with the Taurus object. In Fig. 1 we show the portion of the PSPC image with the X-ray detection of CFHT-BD-Tau 4. In Table 2 we summarize the X-ray parameters of all BDs and BD candidates in Taurus-Auriga, which were detected in at least one PSPC or HRI pointing, i.e. both the objects from SN01 and the new detections.

\section{Discussion}

We compose the largest currently available sample of X-ray emitters near and below the substellar limit by combining all X-ray detections of VLM (spectral type M5 and beyond) objects in star forming regions and young stellar associations, all with ages between 1 and 10 Myrs. In addition to the objects in $\sigma$ Ori and Taurus presented in this paper, X-ray emission has been reported from BDs and BD candidates in Cha I, the Orion Nebular Cluster (ONC), $\rho$ Oph, and IC 348 observed with ROSAT and/or Chandra. According to Neuhäuser \& Comerón (1998) and Comerón et al. (2000) the Cha I star forming cloud hosts seven X-ray emitting BDs and BD candidates. Feigelson et al. (2002) reported about $30 \mathrm{X}$-ray emitting VLM objects in the ONC detected with Chandra. In the $\rho$ Oph star forming region only one BD candidate was found by ROSAT to emit X-rays (Neuhäuser et al. 1999). Recent observations with Chandra by Imanishi et al. (2001) have revealed more X-ray emitting BDs and BD candidates in this region. Furthermore, Preibisch \& Zinnecker (2001) and Preibisch \& Zinnecker (2002) have detected X-ray emission from BDs in IC 348 with Chandra.

In Table 3 we provide the mean X-ray luminosities and $\log \frac{L_{\mathrm{X}}}{L_{\mathrm{bol}}}$ ratios for all objects with spectral type later than M4 in the different star forming regions. The X-ray luminosities of the $\sigma$ Ori objects seem to be somewhat higher than in most other samples. This could be the result of the relatively large distance of the $\sigma$ Ori association, which allows to detect only the X-ray brightest objects with the ROSAT instruments. Note, that at a comparable distance in IC 348 several BDs and BD candidates were detected in a $\sim 50 \mathrm{ksec}$ exposure with Chandra, underlining the value of long observations at high sensitivity in the search for X-ray emission from substellar objects. The most 
Table 1. VLM $\sigma$ Ori members in the error box of a ROSAT source, and further potential optical counterparts from the SIMBAD, GSC or DSS catalogues, respectively. We give object name, X-ray position, ROSAT observation request number, offset between X-ray and optical position of the object in $\sigma$ Ori, optical counterpart from catalogue, and offset between X-ray source and catalogued counterpart. ${ }^{*}$ Note that we use the abbreviations $\sigma$ Ori 053926.8 and $\sigma$ Ori 053948.1 in the text.

\begin{tabular}{|c|c|c|c|c|c|c|}
\hline Designation & $\alpha_{\mathrm{x}(2000)}$ & $\overline{\delta_{\mathrm{x}(2000)}}$ & ROR & $\begin{array}{r}\text { Offset }_{x} \\
{\left[{ }^{\prime \prime}\right]}\end{array}$ & $\overline{G S C, \text { Simbad, } D S S}$ & $\begin{array}{r}\text { Offset }_{\text {cat }} \\
{\left[{ }^{\prime \prime}\right]}\end{array}$ \\
\hline S Ori 03 & 053920.8 & -023035 & $201151 p$ & 0.0 & - & - \\
\hline$\prime \prime$ & 053920.4 & -023036 & $201915 \mathrm{~h}$ & 5.6 & - & - \\
\hline S Ori 08 & 053907.6 & -022824 & $201151 p$ & 23.9 & $D S S$ & $\sim 1.5$ \\
\hline S Ori 51 & 053902.7 & -022955 & $201151 p$ & 26.1 & $D S S$ & $\sim 2.0$ \\
\hline S Ori 07 & 053907.6 & -023237 & $201915 \mathrm{~h}$ & 9.9 & Haro 5-19 & 2.3 \\
\hline S Ori 43 & 053814.0 & -023507 & $201151 p$ & 4.1 & $12906026(\mathrm{GSC})$ & 2.2 \\
\hline 11 & 053813.7 & -023508 & 201915h & 3.9 & 11 & 11 \\
\hline S Ori 053926.8-022614* & 053925.7 & -022612 & $201151 p$ & 17.2 & - & - \\
\hline S Ori 053948.1-022914* & 053948.1 & -022907 & $201151 p$ & 7.1 & - & - \\
\hline
\end{tabular}
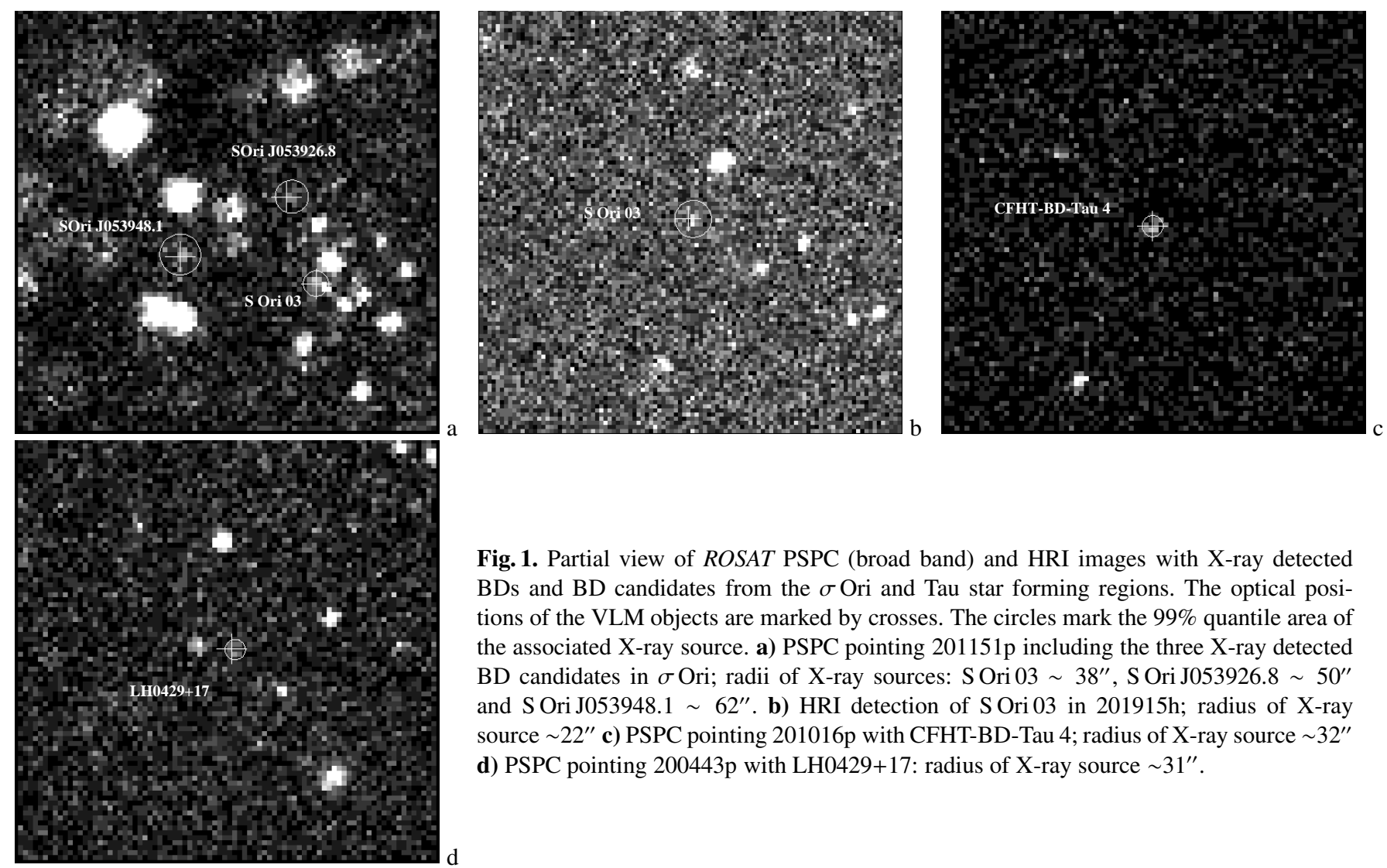

Fig. 1. Partial view of ROSAT PSPC (broad band) and HRI images with X-ray detected BDs and BD candidates from the $\sigma$ Ori and Tau star forming regions. The optical positions of the VLM objects are marked by crosses. The circles mark the $99 \%$ quantile area of the associated X-ray source. a) PSPC pointing 201151p including the three X-ray detected BD candidates in $\sigma$ Ori; radii of X-ray sources: S Ori $03 \sim 38^{\prime \prime}$, S Ori J053926.8 50" and S Ori J053948.1 62". b) HRI detection of S Ori 03 in 201915h; radius of X-ray source $\sim 22^{\prime \prime}$ c) PSPC pointing 201016p with CFHT-BD-Tau 4; radius of X-ray source $\sim 32$ " d) PSPC pointing 200443p with LH0429+17: radius of X-ray source $\sim 31^{\prime \prime}$.

recent X-ray detections of VLM objects in the even more distant ONC were achieved with the Chandra satellite, too. The unusual high value of $\log \frac{L_{\mathrm{x}}}{L_{\mathrm{bol}}}$ for the $\sigma$ Ori objects, however, cannot be explained simply by selection effects and is discussed in the next section.

\section{1. $L_{x}-L_{b o l}-$ relation}

In Fig. 2, we have plotted the X-ray luminosity as a function of the bolometric luminosity for all presently known X-ray detections of BDs and BD candidates in star forming regions. For clarity we omit the large number of non-detections. The dotted and dashed lines mark the range of $\log \frac{L_{\mathrm{x}}}{L_{\mathrm{bol}}}$ from -4.5 to -3 which is typical for T Tauri stars (TTS). According to Fig. 2, objects with masses down to the substellar limit seem to obey a very similar relation although for a few objects $L_{\mathrm{x}}$ is somewhat higher than expected from the canonical relation of latetype stars. From our sample especially three objects, namely LH 0429+17, SOri J053926.8 and SOri J053948.1, lie significantly above the saturation limit of $\log \frac{L_{\mathrm{x}}}{L_{\mathrm{bol}}} \sim-3$ which builds the upper envelope for the X-ray emission from TTS.

The M9-type BD LH 0429+17 was originally listed by Leggett \& Hawkins (1989) as a candidate member of the Hyades cluster. However, both the presence of $\mathrm{Li}$ as an indication of youth and proper motion measurements revealed that it belongs to the Taurus star forming region located in the 
Table 2. X-ray parameters for all ROSAT detected VLM $\sigma$ Ori members and Taurus BD candidates and BDs, sorted by right ascention. The columns contain object name, spectral type, ROSAT observation request number, X-ray position, offset between X-ray position and optical counterpart, offaxis position of the objects within the corresponding FOV, maximum likelihood of existence, exposure time, X-ray counts, X-ray-luminosity, $\log \left(\frac{L_{\mathrm{x}}}{L_{\mathrm{bol}}}\right)$, hardness ratios $H R 1$ and $H R 2$, and the results from the KS-test. The error in the X-ray luminosity does not include the uncertainty in the distance and is discussed in the text.

\begin{tabular}{|c|c|c|c|c|c|c|c|c|c|c|c|c|c|c|}
\hline \multirow[t]{2}{*}{$\overline{\text { Designation }}$} & \multirow[t]{2}{*}{ Sp. type } & \multirow{2}{*}{$\begin{array}{c}R O S A T \\
\text { ROR }\end{array}$} & \multicolumn{2}{|c|}{ X-ray position } & \multirow{2}{*}{$\begin{array}{c}\text { Offset } \\
{\left[{ }^{\prime \prime}\right]}\end{array}$} & $\overline{\text { Offax }}$ & \multirow{2}{*}{\multicolumn{2}{|c|}{$\begin{array}{r}\text { ML Expo } \\
{[\mathrm{ks}]} \\
\end{array}$}} & \multirow[t]{2}{*}{$\overline{\mathrm{Cts}}$} & \multirow{2}{*}{$\begin{array}{l}\log L_{\mathrm{x}} \\
{[\mathrm{erg} / \mathrm{s}]}\end{array}$} & \multirow[t]{2}{*}{$\overline{\log \left(\frac{L_{\mathrm{X}}}{L_{\mathrm{bol}}}\right)}$} & \multirow[t]{2}{*}{ 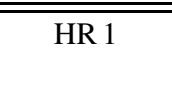 } & \multirow[t]{2}{*}{$\overline{\mathrm{HR} 2}$} & \multirow[t]{2}{*}{$\overline{\text { KS-result }}$} \\
\hline & & & $\alpha_{2000}$ & $\delta_{2000}$ & & {$\left[{ }^{\prime}\right]$} & & & & & & & & \\
\hline \multicolumn{15}{|c|}{$\sigma$ Orionis } \\
\hline$\overline{\text { S Ori03 }}$ & M5-6 ${ }^{a}$ & $201915 \mathrm{~h}$ & 053920.4 & -023036 & 5.6 & 10.8 & 6 & 83.4 & 38.0 & $29.37 \pm 0.16$ & -3.18 & . & 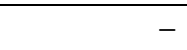 & - \\
\hline 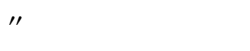 & $"$ & $201151 p$ & 053920.8 & -023035 & $0.0^{b}$ & 10.9 & 59 & 33.4 & 63.5 & $29.53 \pm 0.08$ & -3.02 & $>0.70$ & $0.06 \pm 0.16$ & - \\
\hline SOri J053926.8 & $\mathrm{M}^{a}$ & $201151 p$ & 053925.7 & -022612 & 17.2 & 14.6 & 20 & 31.6 & 31.8 & $29.16 \pm 0.16$ & -1.95 & $>0.99$ & $0.46 \pm 0.22$ & - \\
\hline SOri J & $\mathrm{M}^{a}{ }^{a}$ & $201151 p$ & 053948.1 & -022907 & 7.1 & 17.5 & 42 & 28.9 & 65.0 & $29.54 \pm 0.09$ & -1.49 & $0.69 \pm 0.20$ & $0.30 \pm 0.16$ & - \\
\hline \multicolumn{15}{|c|}{ Taurus } \\
\hline$\overline{\text { FNTau }}$ & M5 & $200949 p$ & 041414.7 & +282754 & 5.4 & 15.6 & 101 & 5.4 & 52.3 & $29.36 \pm 0.15$ & -3.97 & $>0.62$ & $0.31 \pm 0.14$ & $\overline{-}$ \\
\hline RX J0416.5+2053 & M5-6 & $201316 p$ & 041630.2 & +205307 & 38.8 & 24.0 & 9 & 3.1 & 7.3 & $28.74 \pm 0.39$ & $*$ & $>-0.24$ & $0.07 \pm 0.42$ & - \\
\hline$\prime \prime$ & $\prime \prime$ & $201504 p$ & 041631.5 & +205336 & 51.6 & 43.3 & 20 & 5.1 & 20.3 & $28.97 \pm 0.36$ & $*$ & $>-0.69$ & $0.75 \pm 0.22$ & - \\
\hline V410x-ray3 & M6.5 & $001 p-0 / p-1$ & 041808.4 & +282600 & 7.2 & 4.9 & 74 & 28.2 & 57.3 & $28.68 \pm 0.16$ & -3.83 & $>0.33$ & $0.02 \pm 0.16$ & $>99 \%$ \\
\hline StromAnon 13 & M5 & $200001 p-0 / p-1$ & 041818.1 & +282841 & 12.1 & 3.2 & 13 & 30.8 & 9.3 & $27.85 \pm 0.55$ & -4.43 & $>-0.41$ & $0.81 \pm 0.39$ & - \\
\hline Kim3-89 & M5 & $200001 p-0 / p-1$ & 041901.5 & +281944 & 4.1 & 10.3 & 62 & 27.9 & 54.1 & $28.78 \pm 0.10$ & -4.19 & $0.92 \pm 0.26$ & $0.23 \pm 0.17$ & - \\
\hline V410x-ray5a & M5 & $200001 p-0 / p-1$ & 041901.7 & +282233 & 4.1 & 8.6 & 141 & 26.3 & 83.5 & $28.87 \pm 0.15$ & -3.52 & $>0.46$ & $0.35 \pm 0.12$ & - \\
\hline$\prime \prime$ & $\prime \prime$ & $202156 \mathrm{~h}$ & 041902.0 & +282234 & 0.1 & 6.6 & 14 & 7.4 & 15.2 & $29.66 \pm 0.15$ & -2.73 & - & - & - \\
\hline $\mathrm{J} 2-157$ & M5.5 & $200442 p$ & 042053.0 & +174640 & 6.0 & 16.0 & 128 & 18.1 & 133.2 & $29.18 \pm 0.06$ & -3.25 & $0.09 \pm 0.10$ & $0.09 \pm 0.13$ & - \\
\hline$\prime \prime$ & $\prime \prime$ & $201370 p-0 / p-1$ & 042054.3 & +174606 & 42.9 & 39.5 & 13 & 12.6 & 81.4 & $29.14 \pm 0.13$ & -3.29 & $0.16 \pm 0.25$ & $0.26 \pm 0.25$ & - \\
\hline MHO-4 & M5 & $200443 p$ & 043122.9 & +180007 & 21.8 & 32.7 & 13 & 15.2 & 47.5 & $28.91 \pm 0.20$ & -3.28 & $0.54 \pm 0.39$ & $-0.39 \pm 0.26$ & $>99 \%$ \\
\hline$\prime \prime$ & $\prime \prime$ & $201313 p / 900353 p$ & 043124.2 & +180024 & 3.9 & 6.5 & 82 & 11.5 & 39.9 & $29.04 \pm 0.09$ & -3.14 & $>0.66$ & $0.09 \pm 0.17$ & - \\
\hline V927Tau & M5.5 & $200694 p-0 / p-1$ & 043123.6 & +241056 & 2.8 & 21.7 & 62 & 2.6 & 31.5 & $29.51 \pm 0.11$ & -3.77 & $0.61 \pm 0.19$ & $-0.19 \pm 0.20$ & $>98 \%$ \\
\hline MHO-5 & M6 & $201313 p / 900353 p$ & 043215.1 & +181248 & 13.3 & 16.6 & 14 & 9.9 & 12.2 & $28.60 \pm 0.23$ & -3.47 & $>-0.30$ & $-0.08 \pm 0.34$ & - \\
\hline RX J0432.7+1809 & M5 & $201313 p / 900353 p$ & 043240.6 & +180923 & 5.0 & 17.6 & 26 & 7.1 & 17.7 & $28.89 \pm 0.20$ & $*$ & $0.96 \pm 0.49$ & 0.66 & $100 \%$ \\
\hline LH0429+17 & M9 & $200443 p$ & 043250.4 & +173010 & 10.1 & 4.9 & 8 & 20.2 & 18.7 & $28.18 \pm 0.26$ & -2.16 & $-0.24 \pm 0.35$ & $0.58 \pm 0.57$ & - \\
\hline CFHT-BD-Tau 4 & M7 & $201016 p$ & 043947.2 & +260149 & 10.0 & 6.8 & 54 & 10.0 & 28.1 & $28.91 \pm 0.12$ & -3.33 & $0.74 \pm 0.26$ & $0.45 \pm 0.21$ & - \\
\hline
\end{tabular}

${ }^{a}$ Spectral type estimated from colors $I-J, I-K$, and $R-I$, respectively.

${ }^{b}$ No offset between optical and X-ray position as X-ray counts were extracted at the optical position (see text). 
Table 3. Mean X-ray luminosities and $L_{\mathrm{x}} / L_{\text {bol }}$ values for the X-ray detected objects near the substellar limit in different star forming regions. We give also the number of detected sources $\left(N_{\mathrm{D}}\right)$ and the distance.

\begin{tabular}{lrrrllr}
\hline \hline Region & $N_{\mathrm{D}}$ & $\begin{array}{r}\log \left\langle L_{\mathrm{x}}\right\rangle \\
{[\mathrm{erg} / \mathrm{s}]}\end{array}$ & $\begin{array}{r}\log \left\langle\frac{L_{\mathrm{x}}}{L_{\mathrm{bol}}}\right\rangle \\
{[\mathrm{erg} / \mathrm{s}]}\end{array}$ & $\begin{array}{l}\text { dist. } \\
{[\mathrm{pc}]}\end{array}$ & Instr. & Ref. \\
\hline Cha I & 7 & 28.3 & -3.7 & 160 & ROSAT & $(1,2)$ \\
$\rho$ Oph & $1+6$ & 29.2 & -3.7 & 160 & ROSAT + Chandra & $(3,4)$ \\
IC 348 & 7 & 28.2 & -3.5 & 310 & Chandra & $(5)$ \\
ONC & 30 & 29.2 & -2.9 & 470 & Chandra & $(6)$ \\
Tau & 13 & 29.1 & -3.0 & 140 & ROSAT & \\
$\sigma$ Ori & 3 & 29.4 & -2.3 & 350 & ROSAT & \\
\hline
\end{tabular}

(1) Neuhäuser \& Comerón (1998), (2) Comerón et al. (2000), (3) Neuhäuser et al. (1999), (4) Imanishi et al. (2001), (5) Preibisch \& Zinnecker (2001), (6) Feigelson et al. (2002).

background of the Hyades (Reid \& Hawley 1999). If LH 0429+17 were actually associated with the Hyades, and its distance was three times smaller than assumed, this would not shift the object towards the expected relation in Fig. 2, as the values for $\log L_{\mathrm{x}}$ and $\log L_{\mathrm{bol}}$ are both affected in the same direction.

Concerning the $\sigma$ Ori objects we stress that their bolometric luminosity is subject to considerable uncertainty. We based our calculations of the bolometric correction on $I$ band magnitudes. For spectral type M6 and later, we used values for $B C_{\mathrm{I}}$ from Comerón et al. (2000). For earlier spectral types we used the data for main sequence stars given by Kenyon \& Hartmann (1995) which probably come close to those of our pre-main sequence objects, but may not be precise. Further uncertainty derives from the fact that the spectral types for all three detected objects in $\sigma$ Ori were only estimated from their colors, and are uncertain by $\sim 2$ subclasses. As a consequence $B C_{\mathrm{I}}$, and hence $L_{\text {bol }}$ might have been underestimated. Finally, the uncertainty in the distance measurement of the $\sigma$ Ori association is remarkably high. The error of the mean parallax of $\pi=2.84$ mas measured by HIPPARCOS is \pm 0.91 mas, resulting in a distance interval from $267 \mathrm{pc}$ to $518 \mathrm{pc}$. This, however, together with the fact that membership to the cluster is not yet confirmed for all objects of the sample (which would affect their distance) does not result in a change of $\log \frac{L_{\mathrm{x}}}{L_{\mathrm{bol}}}$ as $L_{\mathrm{x}}$ and $L_{\mathrm{bol}}$ are affected in a similar way.

Both for $\sigma$ Ori and Tau, we can also exclude that the unusually high values for $L_{\mathrm{x}}$ have their origin in a flare as the objects under consideration do not show significant variability (see Sect. 5.3). We also checked for indications of circumstellar disks, which would result in a higher extinction and resulting mis-estimate of $L_{\mathrm{x}}$. (Note that it is unclear whether extinction by accretion disks has similar or differing effects on $L_{\mathrm{x}}$ and $L_{\text {bol }}$.) However, $J, H$, and $K$ measurements available from the 2MASS catalogue show no evidence for near infrared excess pointing at the presence of disks in any of the three X-ray detected VLM objects in $\sigma$ Ori. Similarly, from our recently obtained $L$-band photometry of a sample of $\sigma$ Ori BDs - not coincident with the X-ray emitting sample $-K-L$ excess is revealed in only 1 out of 6 examined objects (Jayawardhana et al. 2002).

In the sample in the ONC quite a few VLM objects show significantly higher $\log \frac{L_{\mathrm{x}}}{L_{\mathrm{bol}}}$ ratios than -3 . Together with the $\sigma$ Ori association the ONC belongs to the youngest star forming regions. Therefore, we argue that a high $\log \frac{L_{\mathrm{x}}}{L_{\mathrm{bol}}}$ ratio could be a property of very young low-mass stars and/or BDs.

\subsection{Other activity parameters}

To examine the activity near the substellar boundary in more detail we compared the X-ray emission of the young BDs and $\mathrm{BD}$ candidates to their $\mathrm{H} \alpha$ emission and rotation rates. No rotation periods are known for the objects of our sample. The only published measurements of rotation for young BDs so far are the $v \sin i$ values of six BDs in the Cha I complex, given by Joergens \& Guenther (2001). Those data do not yield any correlation between X-ray activity and $v \sin i$.

The data plotted in Fig. 3 show the relation between $\log L_{\mathrm{x}}$ and the equivalent width of the $\mathrm{H} \alpha$ emission line. The vertical bars show the error in $\log L_{\mathrm{x}}$, but the horizontal bars indicate the range between the lowest and highest value that was measured for the $\mathrm{H} \alpha$ equivalent width. The data suggests that the X-ray luminosity decreases with increasing $\mathrm{H} \alpha$ emission. However, the equivalent width of $\mathrm{H} \alpha$ depends on spectral type, and more universal measures of chromospheric emission such as $L_{\mathrm{H} \alpha}$ are clearly required. The trend shown in Fig. 3 and discussed above reminds of the tendency observed for the (higher-mass) TTS in Taurus, where classical TTS, i.e. those with accretion disks, show stronger $\mathrm{H} \alpha$ but weaker X-ray emission than the diskless weak-line TTS. In this sense $\mathrm{H} \alpha$ emission would probe accretion rather than chromospheric activity.

Most recently, Natta \& Testi (2001) reported that for the BD ChaH $\alpha 1$ (the object with strongest $\mathrm{H} \alpha$ variability in our Fig. 3) the observed IR emission is well described by a circumstellar disk model analogous to that for a TTS. For most of the BDs and BD candidates from the sample shown in Fig. 3 no evidence for disks has been presented yet. But a number of recent studies have shown that some young substellar objects have JHKL excesses (Wilking et al. 1999; Muench et al. 2001) indicative of disks. More attention to the evolutionary status of these objects is clearly needed.

The relation between X-ray luminosity and PSPC hardness ratio $H R 1$ is shown in Fig. 4. The arrows denote upper limits in $H R 1$, i.e. there were no source counts in the soft band, so the maximum number of photons for the $S$-band was extracted from the background. Both the $H R$ 1-ratios and its upper limits indicate that the X-ray emission of the ROSAT detected VLM objects in $\sigma$ Ori and Tau is stronger in the hard band, a sign of strong magnetic activity and youth. A tendency towards larger $H R 1$ for objects with higher $L_{\mathrm{x}}$ is observed, which is in line with results by Preibisch (1997) who showed for a sample of $\mathrm{T}$ Tauri stars that $L_{\mathrm{x}}$ rises with increasing coronal temperature.

In Figs. 5 and 6 we plot $\log L_{\mathrm{x}}$ and $\log \frac{L_{\mathrm{x}}}{L_{\mathrm{bol}}}$ as a function of spectral type. In addition to the VLM objects treated so far we add the sample of X-ray emitting M-type T Tauri stars in the Taurus-Auriga region studied by SN01 from spectral type M0 to M4.5. There is a monotonic decrease in $\log L_{\mathrm{x}}$ towards later spectral types. $\log \frac{L_{\mathrm{x}}}{L_{\mathrm{bol}}}$, however, which is independent of radius and hence of the emitting area, does not change significantly at the transition to substellar masses near spectral type $\sim \mathrm{M} 7$. 


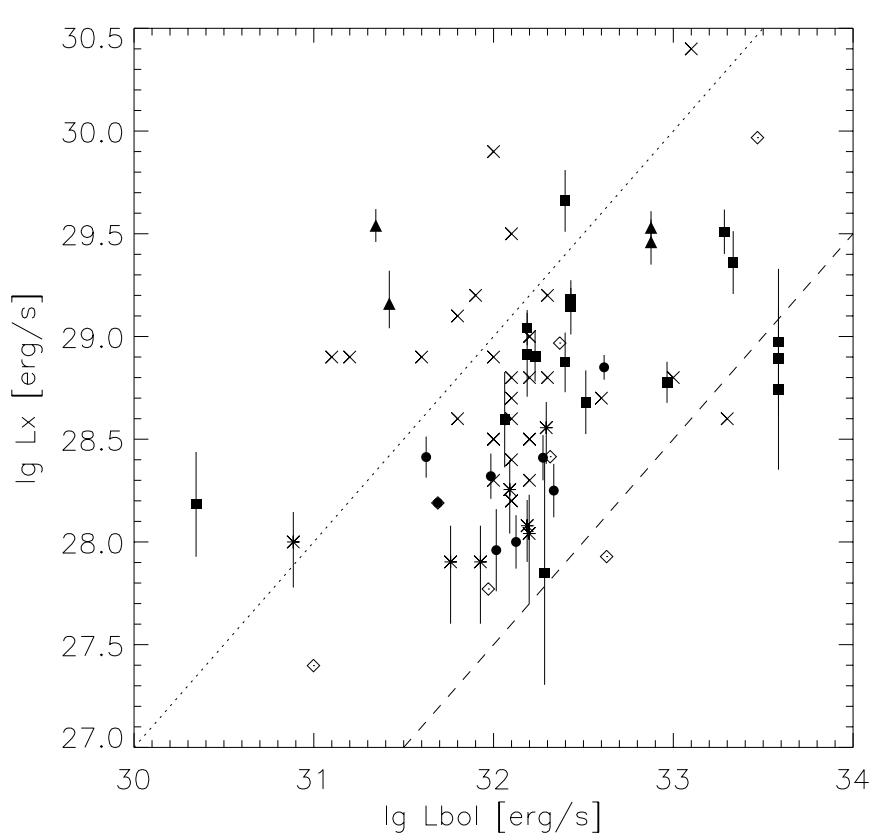

Fig. 2. Correlation between $\log L_{\mathrm{x}}$ and $\log L_{\mathrm{bol}}$ for BDs and BD candidates observed with X-ray satellites: ROSAT observations in TaurusAuriga (squares), Chameleon I (filled circles), $\sigma$ Ori (triangles); Chandra observations in IC 348 (asterisks) and ONC (crosses). $\rho$ Oph was observed both with ROSAT (filled diamond) and Chandra (empty diamonds). The interrupted lines mark the area typical for TTS.

\subsection{X-ray variability}

Visual inspection of the X-ray lightcurves in the sample of Neuhäuser et al. (1999) presented no evidence for variability. Here, we performed a systematic search for variability in all ROSAT detected young BDs and BD candidates making use of the unbinned Kolmogorov-Smirnov (KS) test. We applied the KS-test on the photon arrival times of each source after removing data gaps which are due to the satellite operation. We also checked for variability in the background at a nearby sourcefree region using the same approach. The individual results for the objects in $\sigma$ Orionis and Taurus are given in the last column of Table 2. None of the 6 ROSAT detected BDs and BD candidates in Cha I which are clearly resolved (Comerón et al. 2000) turned out to be variable. The only BD candidate in $\rho$ Oph detected by Neuhäuser et al. (1999) shows no variability either. The total number of objects displaying variability above the $95 \%$ level is 4 (out of 23 examined cases). Preibisch \& Zinnecker (2002) observed variability in only one out of the 7 Chandra detected BDs/BD candidates in IC 348. For a detailed study of variability of the X-ray emission from BDs and BD candidates there is a need of longterm study including longer, continuous observing intervals.

\section{Summary and conclusions}

We have searched for X-ray emission from VLM objects near and below the substellar limit in the young stellar associations $\sigma$ Ori and Tau. Three objects in $\sigma$ Ori and 13 in Tau were detected in ROSAT PSPC and HRI observations. We combined these results with all X-ray detections from BDs and

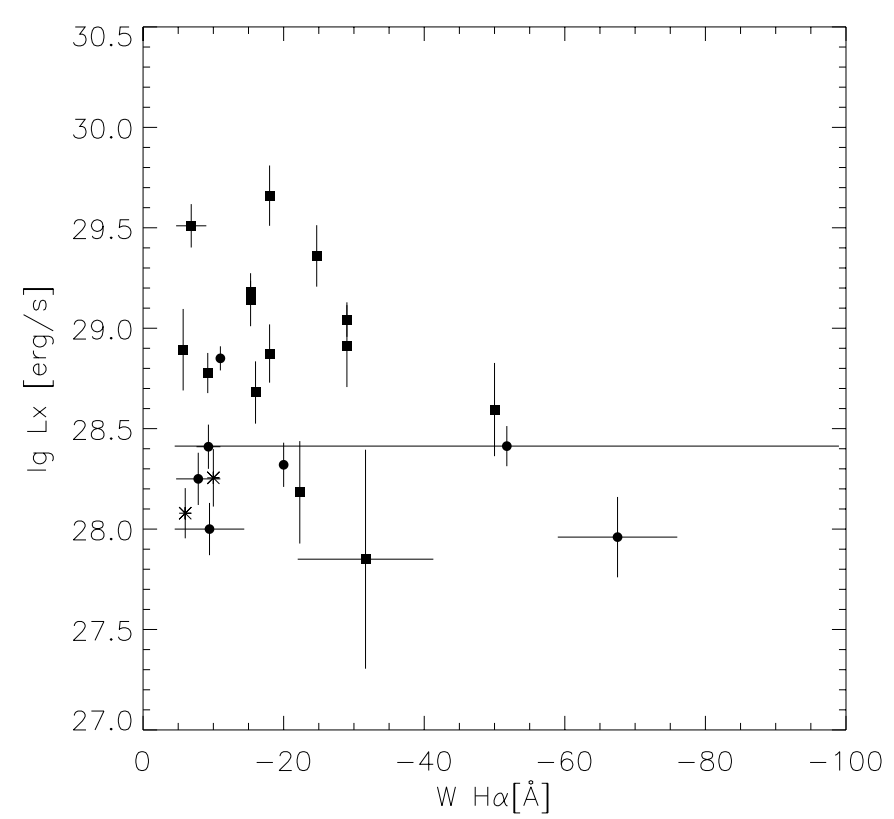

Fig. 3. Relation between $\log L_{\mathrm{x}}$ and $W_{\mathrm{H}_{\alpha}}$ for objects from Fig. 2. This sample, however, is smaller, as $W_{\mathrm{H}_{\alpha}}$ is not known for all objects. Plotting symbols have the same meaning as above. For $W_{\mathrm{H}_{\alpha}}$ measurements see references in Table 3.

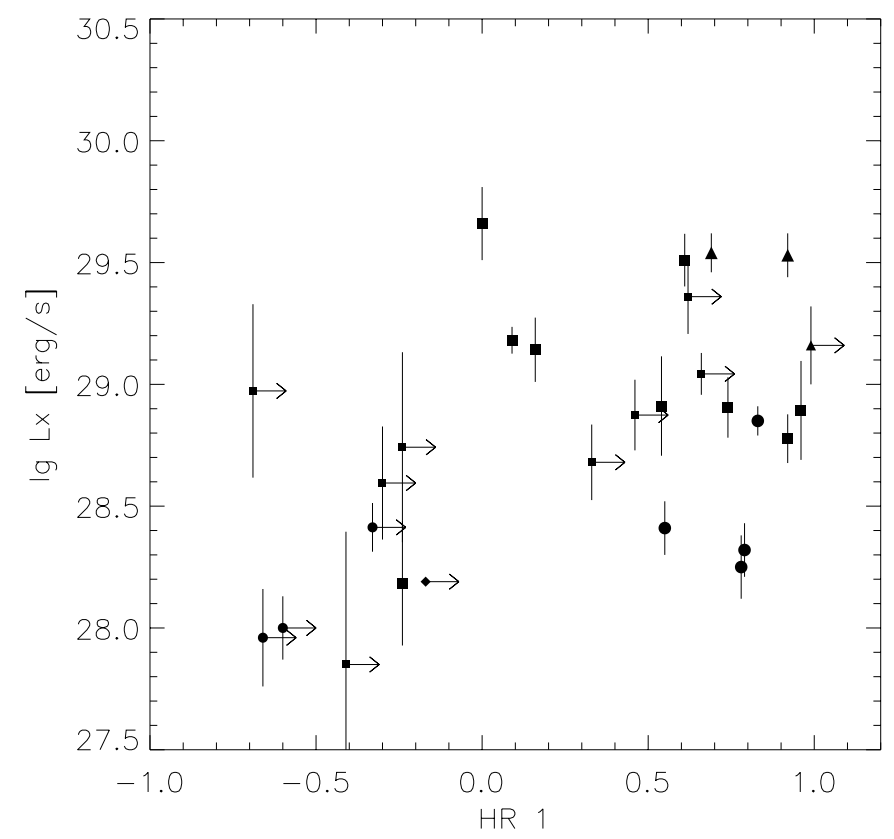

Fig. 4. Relation between $\log L_{\mathrm{x}}$ and $H R 1$ for all ROSAT PSPC detected young BDs and BD candidates. Plotting symbols are defined as in the figures above. Arrows denote upper limit measurements.

BD candidates in star forming regions available up to date and studied stellar activity parameters such as X-ray emission, the ratio $\log \frac{L_{\mathrm{x}}}{L_{\mathrm{bol}}}, \mathrm{H} \alpha$ emission and X-ray hardness ratios of the whole sample. The comparison of the X-ray emission of the young BDs and BD candidates with that of (higher-mass) TTS in Taurus shows that $L_{\mathrm{x}}$ decreases monotonically into the BD regime. However, $\log \frac{L_{\mathrm{x}}}{L_{\mathrm{bol}}}$ remains approximately constant suggesting that the efficiency at which hot coronal gas is produced does not change. For a statistical evaluation of X-ray 


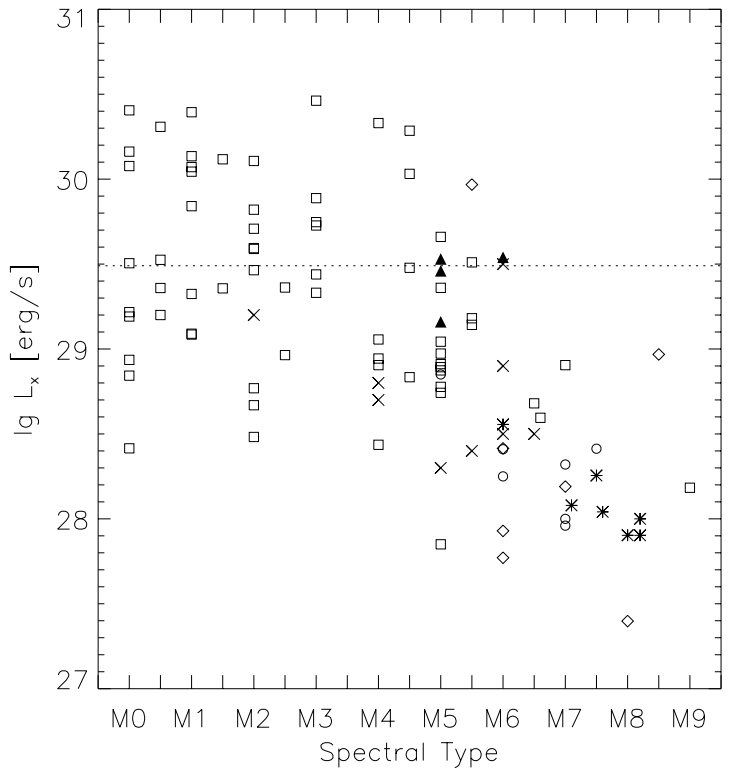

Fig. 5. Relation between $\log L_{\mathrm{x}}$ and spectral type. Symbols have the same meaning as in the figures above. For reasons of clarity, however, we left all symbols empty, except for the $\sigma$ Ori objects. In addition to the BDs and BD candidates discussed in this paper, we added the sample of T Tauri stars in the Taurus-Auriga region from SN01 for comparison. The dashed line denotes the completeness limit for the ROSAT PSPC observation of the $\sigma$ Ori cluster. Note that the spectral types of the $\sigma$ Ori members are only rough values estimated from their colors. For some of the objects in the ONC, spectral types are given as an interval (see Feigelson et al. 2002); in those cases we plot the mean value.

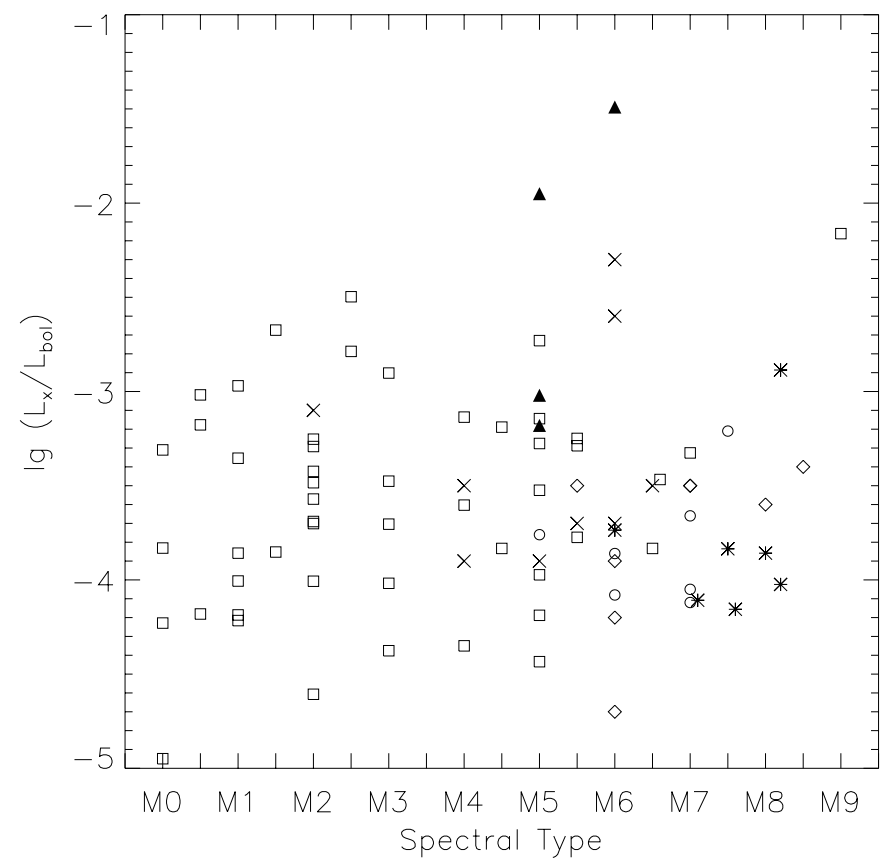

Fig. 6. Relation between $\log \frac{L_{x}}{L_{\mathrm{b}}}$ and spectral type. Symbols have the same meaning as in the figures above.

variability we applied the $\mathrm{KS}$-test to all ROSAT detected BDs and BD candidates in star forming regions. We found that $\sim 17 \%$ of the sources are variable with $>95 \%$ confidence. To complete the study on activity parameters in young
VLM stellar and substellar objects, there is a clear need for rotation and $\mathrm{H} \alpha$ measurements and a search for infrared excess giving clues for the presence of circumstellar disks.

Acknowledgements. We would like to thank R. Neuhäuser for constructive discussions and for reading the manuscript. BS wishes to acknowledge financial support from the BMBF through the DLR under grant number 50-OR-0104. The ROSAT project is supported by the Max-Planck-Gesellschaft and the German federal government (BMBF/DLR).

\section{References}

Barrado y Navascues, D., Zapatero Osorio, M. R., Béjar, V. J. S., et al. 2001, A\&A, 377, L9

Basri, G. 2000, ARA\&A, 38, 485

Basri, G. 2001, in 11th Cambridge Workshop on Cool Stars, Stellar Systems and the Sun, ed. R. J. García Lopez, R., Rebolo, \& M. R. Zapaterio Osorio, ASP Conf. Ser., 223, 261

Béjar, V. J. S., Zapatero Osorio, M. R., \& Rebolo, R. 1999, ApJ, 521, 671

Béjar, V. J. S., Martín, E. L., Zapatero Osorio, M. R., et al. 2001, ApJ, 556,830

Comerón, F., Neuhäuser, R., \& Kaas, A. A. 2000, A\&A, 359, 269

Cruddace, R. G., Hasinger, G. R., \& Schmitt, J. H. 1987, in ESO Conf. Workshop Proc., ed. F. Murtagh, \& A. Heck, 28, 177

Elias, J. H. 1978, ApJ, 224, 857

Feigelson, E. D., \& Nelson, P. I. 1985, ApJ, 293, 192

Feigelson, E. D., Bross, P., Gaffney III, J. A., et al. 2002, ApJ, in press

Fleming, T. A., Schmitt, J. H. M. M., \& Giampapa, M. S. 1995, ApJ, 450, 401

Garmire, G., Feigelson, E. D., Broos, P., et al. 2000, ApJ, 120, 1426

Gizis, J. E., Monet, D. G., Reid, I. N., et al. 2000, AJ, 120, 1085

Imanishi, K., Tsujimoto, M., \& Koyama, K. 2001, ApJ, 563, 361

Jayawardhana, R., Ardila, D., \& Stelzer, B. 2002, in Brown Dwarfs, IAU Symp. 211, ed. E. L. Martín, in press

Joergens, V., \& Guenther, E. 2001, A\&A, 379, L9

Kenyon, S. J., \& Hartmann, L. 1995, ApJS, 101, 117

Lee, T. A. 1968 , AJ, 152, 913

Leggett, S. K., \& Hawkins, M. R. S. 1989, MNRAS, 238, 145

Martín, E. L., Zapatero Osorio, M. R., Barrado y Navascues, D., et al. 2001a, ApJ, 558, L117

Martín, E. L., Dougados, C., Magnier, E., et al. 2001b, ApJ, 561, L195

Mohanty, S., \& Basri, G. 2002 [astro-ph/0201455]

Muench, A. A., Alves, J., Lada, C. J., \& Lada, E. A. 2001, ApJ, 558, L51

Natta, A., \& Testi, L. 2001, A\&A, 372, L22

Neuhäuser, R., Sterzik, M. F., Schmitt, J. H. M. M., Wichmann, R., \& Krautter, J. 1995, A\&A, 297, 391

Neuhäuser, R., \& Comerón, F. 1998, Science, 282, 83

Neuhäuser, R., \& Comerón, F. 1999, A\&A, 350, 612

Neuhäuser, R., Briceño, C., Comerón, F., et al. 1999, A\&A, 343, 883

Preibisch, T. 1997, A\&A, 320, 525

Preibisch, T., \& Zinnecker, H. 2001, AJ, 122, 866

Preibisch, T., \& Zinnecker, H. 2002, AJ, 123, 1613

Reid, I. N., \& Hawley, S. L. 1999, AJ, 117, 343

Rutledge, R. E., Basri, G., Martín, E. L., \& Bildsten, L. 2000, ApJ, 538, L141

Stelzer, B., \& Neuhäuser, R. 2001, A\&A, 377, 538 (SN01)

Walter, F. M. 1997, Mem. Sai., 68, 1081

Wilking, B. A., Greene, T. P., \& Meyer, M. R. 1999, AJ, 117, 469

Zapatero Osorio, M. R., Béjar, V. J. S., Martín, E. L., et al. 2000, Science, 290, 103

Zapatero Osorio, M. R., Béjar, V. J. S., Pavlenko, Ya., et al. 2002, A\&A, 384, 937 